\title{
Appointment Template Redesign in a Women's Health Clinic Using Clinical Constraints to Improve Service Quality and Efficiency
}

Y. Huang ${ }^{1}$; S. Verduzco

${ }^{1}$ Department of Industrial Engineering, New Mexico State University, Las Cruces, NM, USA

\section{Keywords}

Appointment scheduling, appointment template, reclassification, clinical constraints

\section{Summary}

Background: Patient wait time is a critical element of access to care that has long been recognized as a major problem in modern outpatient health care delivery systems. It impacts patient and medical staff productivity, stress, quality and efficiency of medical care, as well as health-care cost and availability.

Objectives: This study was conducted in a Women's Health Clinic. The objective was to improve clinic service quality by redesigning patient appointment template using the clinical constraints. Methods: The proposed scheduling template consisted of two key elements: the redesign of appointment types and the determination of the length of time slots using defined constraints. The reclassification technique was used for the redesign of appointment visit types to capture service variation for scheduling purposes. Then, the appointment length was determined by incorporating clinic constraints or goals, such as patient wait time, physician idle time, overtime, finish time, lunch hours, when the last appointment was scheduled, and the desired number of appointment slots, to converge the optimal length of appointment slots for each visit type.

Results: The redesigned template was implemented and the results indicated a $73 \%$ reduction in average patient waiting from the reported 40 to 11 minutes. The patient no-show rate was reduced by $4 \%$ from $24 \%$ to $20 \%$. The morning section on average finished about $11: 50 \mathrm{am}$. The clinic day was finished around 4:45 pm. Provider average idle time was estimated to be about 5 minutes, which can be used for charting/documenting patients.

Conclusions: This study provided an alternative method of redesigning appointment scheduling templates using only the clinical constraints rather than the traditional way that required an objective function. This paper also documented the employed methods step by step in a real clinic setting. The implementation results concluded a significant improvement on patient wait time and noshow rate.

\section{Correspondence to:}

Yu-Li Huang, Ph.D.

Department of Industrial Engineering

MSC 4230, New Mexico State University

P.O. Box 30001

Las Cruces, NM 88003 USA

Email:yhuang@nmsu.edu

\author{
Appl Clin Inform 2015; 6: 271-287 \\ http://dx.doi.org/10.4338/ACl-2014-10-RA-0094 \\ received: October 23, 2014 \\ accepted: March 1, 2015 \\ published: April 22, 2015 \\ Citation: Huang Y, Verduzco S. Appointment Template \\ Redesign in a Women's Health Clinic Using Clinical \\ Constraints to Improve Service Quality and Efficiency. \\ Appl Clin Inf 2015; 6: 271-287 \\ http://dx.doi.org/10.4338/ACI-2014-10-RA-0094
}




\section{Background}

Patient wait time is a critical element of access to care that has long been recognized as a major problem in modern outpatient health care delivery systems. Especially the perception of long waiting in a clinic has been identified as a contributing factor of patients not showing up for their appointments [1-2]. It impacts patient and medical staff productivity, stress, service quality and efficiency of medical care, as well as health-care cost and availability. Many appointment scheduling methods have been developed to undertake the problems with consideration of patient classifications [3-6], appointment demand uncertainty [7-9], urgent care [10-12], patient arrival patterns [13], service interruptions and physician lateness [14], walk-ins [15], ancillary services [16], and no-shows for effective overbooking [17-23]. These considerations aim to provide a better appointment scheduling template by mainly determining the most appropriate appointment intervals in subject to an objective function that either maximizing profit $[8,24-25]$, minimizing patient and/or physician waiting $[13-14,16,19,26-28]$, or minimizing costs of patient waiting, physician idling and overtime [3, 10-11, 15, 17-18, 20-21, 29-30]. However, the costs relative to patient wait time and physician idle time cannot be easily quantified and the cost of physician idle time tends to be much higher than that of patient wait time due to the perception of higher compensation on physicians [31-32], which inherently favors the reduction of physician idle time rather than patient wait time. On the other hand, one cannot assume the effects of patient wait time and physician idle time on clinic flow are equal when considering only waiting. Even though these approaches create a patient-centric appointment system, they tend to overlook availability of medical resources such as physician's clinic preference and clinic policies which may lead to limited patient access to care [27-28]. The open access scheduling method, using the philosophy of "doing today's work today", aims to improve patient access and reduce the no-show rate [33-37], but allows overtime and results in significant patient wait time when the demand is high [19]. This approach increases the cost for clinics and burdens clinics' management.

\section{Objectives}

To avoid the conjecture of the deviation from choosing the right objective function, this research proposed to converge the most appropriate length of appointment slot for each patient group by using only constraints or goals that were defined by a clinic management team without an objective function. The study methods included collecting provider treatment time data, reclassifying patient visit groups, simulating clinic patient flow, defining clinical constraints, determining the most appropriate length of appointment slot for each patient group, and implementing the solution with actual implementation results.

\section{Methods}

The proposed methods were demonstrated in a Women's Health clinic. This scheduling template redesign included provider treatment time data collection, patient visit group reclassification, and appointment slot length determination for provider and patient arrival schedules.

\subsection{Women's Health Clinic}

There were a total of seven fulltime Certified Nurse-Midwives (CNM) in this clinic and all clinic days were covered by at least two CNMs. The clinic currently has eight visit types. They were: Follow-up (FU) - any patients scheduled for review of laboratory results; Birth Control Management (BCM) - any patients scheduled for general birth control counseling; New Obstetric (NOB) - any patients scheduled for her initial OB appointment at the clinic; Routine Obstetric (ROB) - any patients returning for scheduled routine prenatal care appointment; Physical Exam (PE) - any patients scheduled for her annual or semi-annual physical exam; Postpartum (PP) - any patients scheduled for routine scheduled postpartum examination; Level 1 - any patients scheduled for the evaluation 
of pelvic pain, symptoms related to vaginitis, sexually transmitted infection, urinary tract infection, cold, muscle sprains, complaints about intra-uterine device (IUD) use and removal, etc; Level 2 any patient scheduled for the evaluation of high risk pregnancy, initial insertion of IUD, removal of sub-dermal contraceptive implant, colposcopy, cryosurgery, or endometrial biopsy. Given the patient needs from the calendar year of 2012, the distribution of appointment slots per CNM per clinic day was: seven ROB, two PE, one FU, one NOB, one PP, one Level 1, one Level 2, and one BCM. The total demand outlined was for a minimum of 15 appointment slots divided across eight hours based on the level of patient care acuity and expected time commitment for each CNM to complete the scheduled visit in a timeframe that promoted patient satisfaction while increasing provider efficiency at the same time. Therefore, the current schedule was simply in a 30-minute slot scheduling system. However, the current expectation outlined by management was 28 patients per CNM daily, as this assumption was weighed against a high rate of patients who ultimately did not show up for their scheduled appointments. The current no-show rate was around $24 \%$, which in terms of actual patients seen for each CNM in a clinic day was about 22 patients. In order to reach the expected productivity (as measured in terms of revenue generated via CNM led patient encounters in the clinic setting), the current scheduling template was allowed to be stacked to account for the high rate of no-shows which created an environment where patients were routinely double-booked, or scheduled appointment times were separated by one minute intervals. This decision caused the patient wait time to be around 40 minutes on average. The clinic believed that 1) streamlining clinic flow by reworking the scheduling template would increase revenue and patient satisfaction, and 2) reducing current no-show rate to be around $15 \%$. Hence, the research team was asked to redesign a scheduling template based on a $15 \%$ no-show rate.

\subsection{Current Scheduling Issues}

The problems of the current scheduling system were: 1. A 30-minute slot interval was used for all eight patient types. The 30-minute slot may or may not be enough for the time actually needed for CNM to complete a patient; 2 . An observation of clinic flow indicated that CNMs had a tendency to wait for patients to be ready. Some activities were required of patients after check in and before seen by a CNM, such as changing to an exam gown, providing specimens, and taking basic vital signs. The current schedule assumed that the time of appointment was the time of being seen by CNMs and the time of a patient should arrive; 3 . The current overbooking or double-booking policy increased patient wait time significantly. To redesign the current template, the following steps were critical:

1. Collect $\mathrm{CNM}$ actual treatment time for each patient type to understand the actual face to face time a CNM spent with a patient and the frequency of each patient type;

2. Reclassify current patient groups into groups for scheduling purpose that captured the average and variance of CNM treatment time;

3. Build a simulation model that represented the current patient flow that allowed us to test different scheduling options;

4. Define the clinical constraints that agreed by clinic management and medical staff;

5. Use these clinical constraints to converge on a solution for the CNM schedule that met all specified constraints/goals;

6. Determine the length of time required for the pre-visit activities for the patient arrival schedule;

7. Implement the new scheduling template.

\subsection{CNM Treatment Time Data Collection}

The data was collected by the CNMs for a period of two weeks for their actual treatment time with patients. There were a total of seven CNMs with an average of two CNMs work during a clinic day. The data was summarized in $>$ Table 1 . The current amount of time that a patient required from a CNM on average was 14.3 minutes for BCM, 16.1 minutes for FU, 13 minutes for ROB, 25 minutes for Level 1, 26.1 minutes for PP, 37.1 minutes for Level 2, 37.1 minutes for NOB, and 33.7 minutes for PE. The overall average treatment time was about 22 minutes with a standard deviation of 12.6 minutes. The data summary indicated that the current 30-minute slot was designed to accommo- 
date patient treatment time variations, which had the coefficient of variation $(\mathrm{CV})$ of 0.58 . CV showed the extent of treatment time variability in relation to the average of the treatment time. The calculation was standard deviation divided by average of the treatment time; see $>$ Table 1 .

\subsection{Reclassification Patient Groups}

For scheduling purposes, the template was designed to capture the treatment time and its variation. The current 30-minute slot was designed to capture the coefficient of variation (CV) of 0.58 . The goal was to reclassify patients into groups that had a CV less than 0.58 . Researchers indicated that a scheduling system would perform more cost-effectively if a scheduling group had the minimal treatment variation [5]. There were eight patient types in this clinic. It was not feasible in terms of appointment flexibility for a scheduling template to have eight different appointment slots. A reclassification was conducted to regroup patient types. An analysis was carried out to determine the new groups; see $>$ Figure 1. Figure 1 indicated that there was a clear three-group formation based on a 95\% confidence interval. The first group was called Low group (L) that consisted of BCM, FU, and ROB patient types. The second group was called Medium group (M) that consisted of Level 1 and PP. The last group was called High group $(\mathrm{H})$ that consisted of Level 2, NOB, and PP. These new classifiers of $\mathrm{L}, \mathrm{M}$, and $\mathrm{H}$ were used for scheduling purposes. The summary of the new groups was shown in Table 1 . Table 1 concluded that the new L group had a mean treatment time of 14.2 minutes and a standard deviation (SD) of 6.3 minutes, the new M group had a mean of 25.4 minutes and a SD of 9 minutes, and the new $\mathrm{H}$ group had a mean of 35.7 minutes and a SD of 11.2 minutes. The coefficients of variation (CV) were calculated to be $0.44,0.35$, and 0.31 , respectively. This indicated that each of these new groups introduced a lesser CV than 0.58 . In theory, the new scheduling template should outperform the current schedule.

\subsection{CNM Schedule Development}

Three steps were taken in order to finalize the CNM schedule:

1. Determine the number of slots needed for each new patient group;

2. Build a simulation model that reflects the current patient flow for evaluating scheduling options;

3. Decide the most appropriate CNM schedule design considering the clinical constraints.

The minimum volume was set by clinic management based on the past year patient volume with anticipated growth; see $>$ Table 2. Table 2 summarized the minimum volume set based on the current patient type per clinic week into a new patient type. The distributions for the new patient groups were $62.8 \%$ for L patient group, $11.5 \%$ for $\mathrm{M}$ patient group, and $25.6 \%$ for $\mathrm{H}$ patient group.

The next step was to evaluate the performance of each scheduling option. The scheduling options were in terms of the total appointment slots. Four options were evaluated: 22, 24, 26, and 28 patient slots. The distribution percentage of the new patient groups was applied to the 22-slot schedule. Two, four, and six additional slots for 24-slot, 26-slot, and 28-slot schedules respectively were for L group since the management believes that Routine Obstetric (ROB) patients have been growing over the years. Therefore, based on the distribution from $>$ Table 2, the 22-slot schedule consisted of 14 $(22 \times 62.8 \%)$ L patient slots, $2(22 \times 11.5 \%)$ M patient slots, and $6(22 \times 25.6 \%) \mathrm{H}$ patient slots. The 24-slot schedule included $16 \mathrm{~L}$ slots, $2 \mathrm{M}$ slots, and $6 \mathrm{H}$ slots. The 26 -slot schedule had $18 \mathrm{~L}$ slots, 2 $\mathrm{M}$ slots, and $6 \mathrm{H}$ slots. The 28-slot schedule had $20 \mathrm{~L}$ slots, $2 \mathrm{M}$ slots, and $6 \mathrm{H}$ slots. The appointment slots for each patient group were distributed evenly throughout a clinic day indicated by the clinic; see Table 3. Table 3 also indicated a break for lunch in the middle of the clinic day.

Once the schedule options were determined, the simulations of clinic flow were performed. The simulation assumptions were: patient punctual arrival, no-show rate of 15\%, CNM starting on-time, and Gamma distribution for treatment time of each new patient group, since it was used by many researchers as a general service time distribution [16-17, 28, 32]. The length of each appointment slot was evaluated at eleven different levels. Each level was differed by the number of treatment time standard deviation that was accounted for. This number was called "factor" in the study. The eleven factors were $-0.5,-0.4,-0.3,-0.2,-0.1,0,0.1,0.2,0.3,0.4$, and 0.5 . Therefore, the length of an appointment slot was calculated by the average of treatment time plus the factor multiplied by the 
standard deviation of treatment time. For example, given a factor of 0.1 , an $\mathrm{L}$ group patient slot length should be $14.2+0.1 \times 6.3=14.8$ minutes. For another example, if a factor is -0.4 , an $\mathrm{H}$ group slot length should be $35.7+(-0.4) \times 11.2=31.2$ minutes. The performance was measured by average patient wait time (APW), average CNM idle time (ACI), lunch hour (LH), finish time (FT), overtime (OT), last patient scheduled in the morning (LSM), and last patient scheduled in the afternoon (LSA). 100 runs were simulated to summarize these performances. Each run represented a possible clinic day.

After consulting with clinic management regarding the performance level the clinic would like to achieve, the following constraints/goals were obtained:

- Average patient wait time (APW) was 15 minutes or less.

- Average CNM idle time (ACI) or break time in between patients was 3 minutes or less.

- The preference on lunch hours $(\mathrm{LH})$ was at least 45 minutes.

- The preferred overtime (OT) was 10 minutes or less by the end of a clinic day.

- The finish time (FT) in a clinic day was at 5:10 pm (550 minutes for an 8-hour day with one hour lunch break).

- The preferred schedule time for the last patient was 11:45 am (225 minutes) in the morning (LSM) and 4:45 pm (525 minutes) in the afternoon (LSA).

To be noted, most of these constraints were based on the CNMs' preferences and management's goals. There was not a policy in place before this study and this was the first time for this clinic to actually explore their goals operationally. The constraints were considered one at a time to find the associated possible solutions. Figure 2 summarized the performance measures for appointment slot options $(22,24,26$, and 28 slots) versus the length of appointment slots calculated by eleven factors. The possible solutions were highlighted. For a 22-slot schedule, the solutions that satisfied all constraints were factors $-0.2,-0.3,-0.4$, and -0.5 . The corresponding solution sets were for the length of appointment slots in minutes for each new group $(\mathrm{L}, \mathrm{M}, \mathrm{H})$, they are $(12.9,23.6,33.5),(12.3,22.7$, $32.3),(11.7,21.8,31.2)$, and $(11.1,20.9,30.1)$. For a 24 -slot schedule, the solutions that satisfied all constraints were factors $-0.3,-0.4$, and -0.5 , which translated to the solution sets for the length of appointment slots to be $(12.3,22.7,32.3),(11.7,21.8,31.2)$, and $(11.1,20.9,30.1)$. For a 26-slot schedule, the solution was only from a factor of -0.4. Hence, the only solution set was $(11.7,21.8$, 31.2). For a 28 -slot schedule, given the current constraints, there was no solution. After presenting the possible solutions to the clinic management, the clinic decided to go with the solution for 26 slots, which was the closest to the expected target volume outlined by management without overbooking and double-booking. The expected total patients seen for a $15 \%$ no-show rate was about 22 , which was the volume of patients seen currently. Therefore, the CNM schedule was determined; see - Table 4. For example, if the first patient was scheduled 11.7 minutes for L with CNM at 8:00 am, then the second patient should be scheduled at 8:12 am $(0+11.7=11.7)$. If the second patient was also scheduled for $\mathrm{L}$ and it takes about 11.7 minutes, then the third patient should be scheduled at $8: 23$ am $(11.7+11.7=23.4)$. If the third patient was for $\mathrm{H}$ group and the scheduled time should be 31.2 minutes, then the fourth patient should be scheduled at 8:55 am $(23.4+31.2=54.6)$.

\subsection{Patient Arrival Schedule Development}

After CNM schedule was determined, the patient arrival was scheduled accordingly. To understand how long it took for a patient to be ready for CNM, the pre-activities and times were estimated by check-in clerks and medical assistants (MA) /nurses (RN). $>$ Table 5 documented the estimation. For the L group, the maximum time needed for check-in and time needed with MA/RN was 20 minutes. For the M group, the maximum time needed for check-in and time with MA/RN was 30 minutes, since the maximum time for PP was 30 minutes and for Level 1 was 20 minutes. For the $\mathrm{H}$ group, the maximum time needed for check-in and MA/RN time was 30 minutes, since the maximum time for PE was 30 minutes, for Level 2 was 25 minutes, and for NOB was 30 minutes. Then considering the weight of each new group, the average of these maximum times was $20 \times 62.8 \%+30$ $\times 11.5 \%+30 \times 25.6 \% \approx 24$ minutes and it was rounded to be 25 minutes. These 25 minutes were then incorporated into CNM schedule; see $>$ Table 4 . For example, if the first patient was scheduled with CNM at 8:00 am, then the patient should arrive at 7:35 am which was 25 minutes early. If the 
second patient was scheduled with $\mathrm{CNM}$ at 8:12 am, then the patient should arrive at 7:47 am which was rounded to 7:50 am. Hence, for a schedule with CNM at 2:28 pm, the patient should arrive at 2:03 pm, rounded to $2: 05 \mathrm{pm}$.

\section{Results}

The new scheduling scheme was communicated to the front desk especially the schedulers. The training was very minor since the schedulers had had the knowledge of identifying the original eight patient visit types. The only changes were to place these eight visit types into the new scheduling visit types of low, median, and high. The schedulers expressed the change was very simple to adapt. In addition, the upcoming patients were informed the new schedule implementation and encouraged to make their appointments on time. This 26-slot appointment was employed into the system and the data was collected to verify effectiveness. The data collection sheet included arrival time, check-in time, called back time, in room time, appointment time, CNM time, appointment information, CNM in time, and CNM out time. This was a manual process. All parties involved in the process had to write the time based on a digital clock. After one month of data collection, there were a total of 1,042 appointments booked and 838 appointments seen for an average of two CNMs in a clinic day. The results indicated a significant improvement, both on average patient wait time and no-show rate. The average patient wait time was reduced by $73 \%$ from the reported 40 minutes to 11 minutes. Among these 11 minutes, an average of 2 minutes were from the preparation activities and the average of 9 minutes were the wait time for CNMs. The average no-show rate was reduced by $4 \%$ from the initial $24 \%$ to the $20 \%$ during the implementation. The morning section on average finished about 11:50 am. The worst case finished at 12:01 am. The clinic day was finished around 4:45 pm on average. The worst case finished at 5:34 pm and the next worst case finished at 5:13 pm. $\mathrm{CNM}$ average idle time was estimated to be about 5 minutes, which could be used for charting/ documenting patients. The results seemed to reflect quite closely with what simulations predicted and prove that the new scheduling template actually worked to meet most of the target values. The simulations reported the average patient wait time was 13.3 minutes and CNM idle time was 1.9 minutes. The slight difference between actual and simulated data was mostly due to the deviation of the manual data collection process and the simulation assumptions of patient punctual arrival and CNM starting on time. The implementation was very successful. Comments from CNMs were very positive and four of them were:

- "There have been some growing pains, but overall we are now seeing shorter wait times and a much-improved flow in the clinic."

- "The new template is so much better than the old that I welcome the transition!"

- "The template was working beautifully"

- "The template seems to be working well."

Although patients were not interviewed about their experience with this new change, some feedbacks from patients indicated the shorter wait time had made their live much easier. The most critical observation from the implementation data was the actual no-show rate. Due to the communication of implementing the new scheduling system to patients, the no-show rate was reduced by $4 \%$. However, the intended no-show rate was $15 \%$ for the new design, which was $5 \%$ higher. This explains the reasons that patient wait time was two minutes less and CNM idle was about three minutes more. The actual number of patients seen during the implementation was about three patients ((425-419)/2) less per clinic week due to the $20 \%$ no-show rate. The number 425 was from the initial data collection for two weeks with $24 \%$ no-show rate and 419 was from the implementation data collection for a month with $20 \%$ no-show rate translating into two weeks. If the clinic could achieve the intended no-show rate of $15 \%$ with the new schedule, the expected patients seen in two weeks should be about 443 , which was 18 patients more than the old schedule. To reach the target of $15 \%$ no-show rate, the clinic has indicated a need to understand other factors that contribute to the reasons for no-shows. 


\section{Discussions}

From the implementation, we learned the key discrepancy between the actual results and the simulation results was the actual no-show rate. The study team took the initiative and provided possible scheduling solutions for a $20 \%$ no-show rate. Figure 3 summarized the performances and the possible solutions. With $20 \%$ no-show rate, the clinic could actually have a 28 -slot appointment (expected by clinic management) given the same target values of average patient wait time, average CNM idle time, overtime, finish time, lunch hour, and the scheduled time for the last patient in the morning and afternoon. The 28-slot solution was to schedule the L group at 11.1 minutes, the M group at 20.9 minutes, and the $\mathrm{H}$ group at 30.1 minutes with a factor of -0.5 . This solution was expected to reduce patient wait time by $65 \%$ from 40 minutes to about 14 minutes.

The research team also ran simulations to demonstrate the variation from the choice the clinic made regarding the sequence of patient type scheduled in a clinic day. The current choice was to distribute each patient type evenly throughout a clinic section (morning or afternoon section), which was believed that it provided better options for different patient types [28]. However, some researchers demonstrated that the sequence of heterogeneous patient types could have a more drastic effect on schedule performance [38-39]. The scheduling sequence of placing large service time standard deviations at the end of each section was shown in - Figure 4. The performance for 22, 24, 26, and 28 slots was also summarized in $>$ Figure 4 . The results indicated that there was still no solution for the 28-slot scenario, but three solutions for the 26-slot and the performance in terms of patient wait time, physician idle time, overtime, the finish time, lunch hour, the last patient scheduled for both morning and afternoon sections was improved. For example, for the 26-slot given the factor of -0.4 , the solution that placed patient types in order of $\mathrm{L}, \mathrm{M}$, and $\mathrm{H}$ in a session compared to the solution that converged from the sequence that distributed each patient evenly throughout a session had reduced patient wait time by $16 \%$, physician idle time by $9 \%$, overtime by $10 \%$, and 4 minutes in finish time. It had also increased 4 minutes for lunch hour and was able to schedule the last patient 20 minutes earlier in both sessions. This discussion provided the clinic an alternative scheduling sequence that consequently improved clinic service performance.

\section{Conclusions}

This study provided an alternative method of redesigning appointment scheduling templates using the clinical constraints only rather than the traditional way that required an objective function. This study also documented the employed methods step by step to demonstrate the feasibility in a real clinic setting including collecting provider treatment time data, reclassifying patient groups for scheduling purpose, simulating clinic patient flow to trial various scheduling options, defining clinic performance constraints/goals with management team, and summarizing possible scheduling options for the clinic. The clinic performance constraints could include, but not limited to, average patient wait time, average physician idle time, overtime, finish time, lunch hours, when the last appointment was scheduled, and the desired number of appointment slots in a clinic day to converge the optimal length of appointment slot for each visit type. The implementation of the participating Women's Health Clinic resulted in a significant $73 \%$ reduction on patient wait time from 40 minutes to 11 minutes on average and $4 \%$ reduction on patient no-show rate from $24 \%$ to $20 \%$. The research team believed that the $4 \%$ no-show rate reduction could be the results of communication with patients regarding the efforts of scheduling template redesign to improve service quality. The new template was intended to have a no-show rate of $15 \%$ to reach its maximum potential. If the no-show rate continued declining to be below 15\%, a new template using the proposed method would be necessary in order to maintain clinic constraints and service quality. However, if the no-show rate over time stayed above $15 \%$, the clinic needed to investigate the reasons for patient no-shows. As the results section indicated, some of the clinic flow disruptions came from the patient on-time arrival, the provider on-time treatment, and the patient no-shows. These issues are potential research for policy changes such as providing incentives to both patients and providers. In the case where a clinic is not comfortable issuing the incentive systems, the concerns will have to be accounted for when 
redesigning the appointment template in the simulation modeling stage, which their impact on scheduling templates can be further discussed in our immediate future research.

\section{Clinical Relevance Statement}

This study aimed to reduce patient wait time using clinical constraints and goals without any objective function in a Women's Health Clinic. The step-by-step methods discussed could guide and assist other clinics to redesign their appointment templates to be more patient-centric and efficient. The implementation results further demonstrated the feasibility of the proposed methods.

\section{Conflicts of interest statement}

The authors declare that they have no conflicts of interest in the research.

\section{Protection of Human and Animal Subjects}

The research study was not human subject research. 


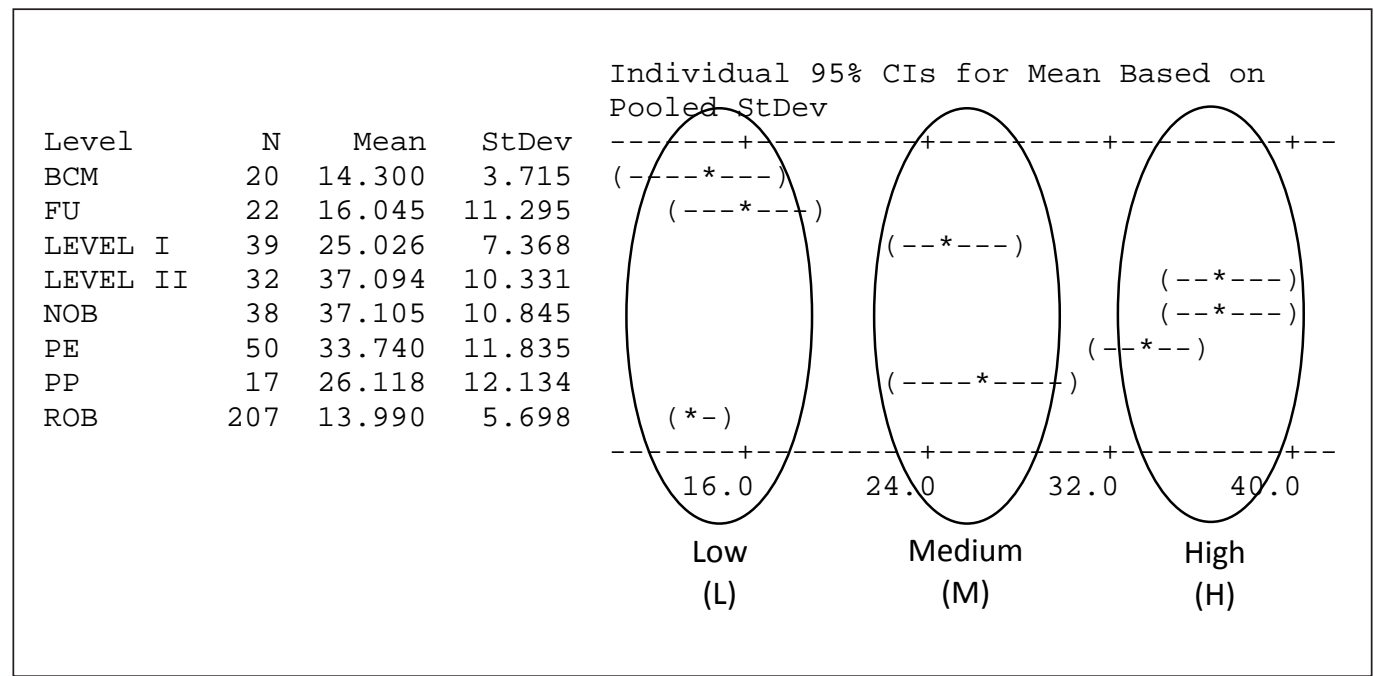

Fig. 1 Reclassification of patient groups 


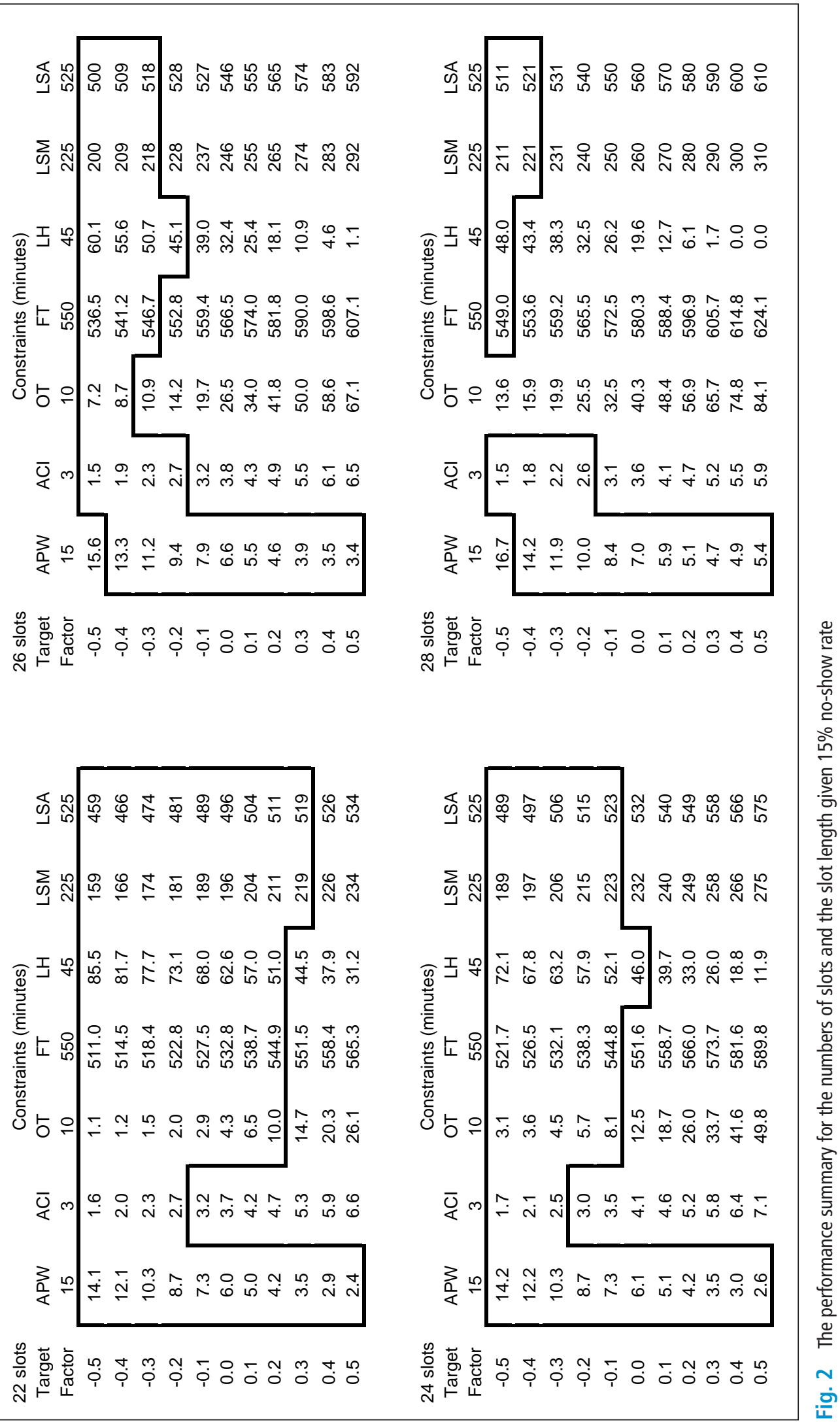




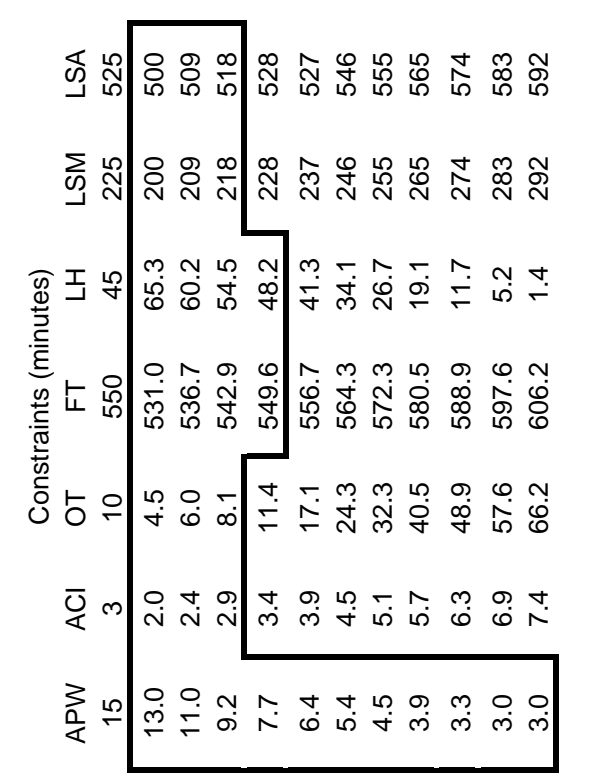

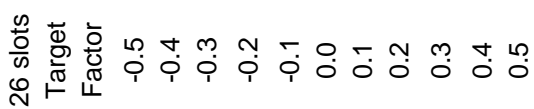

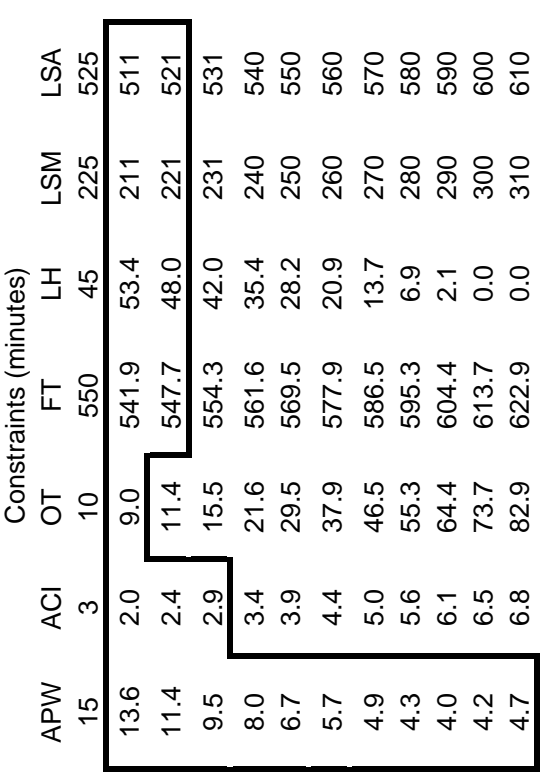

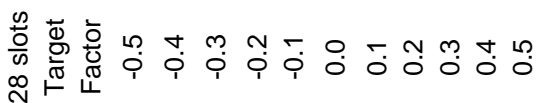

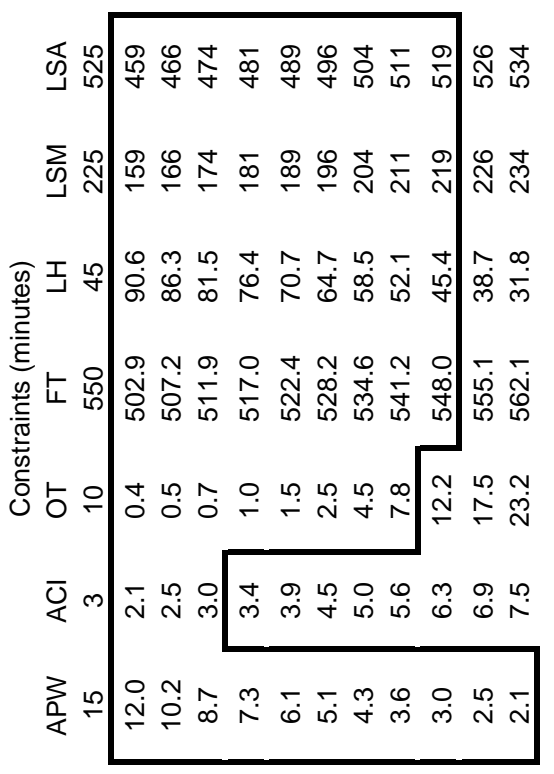

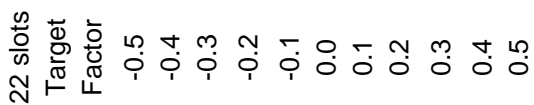

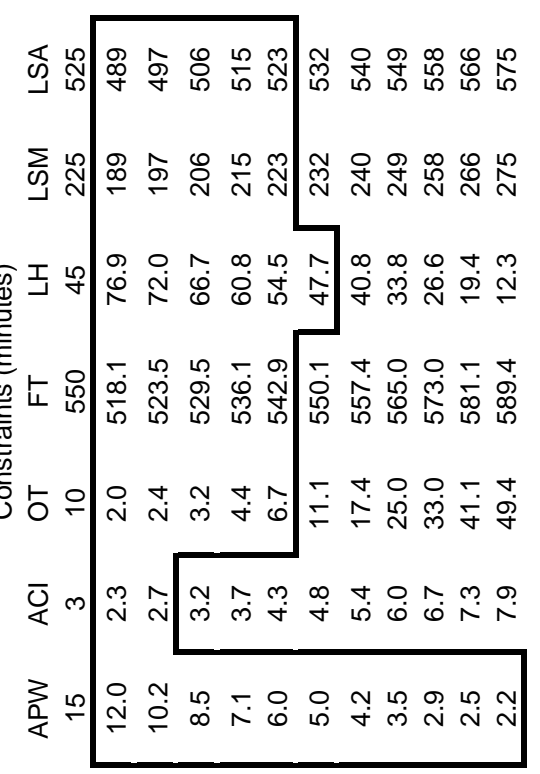

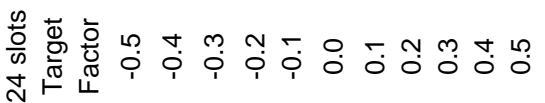




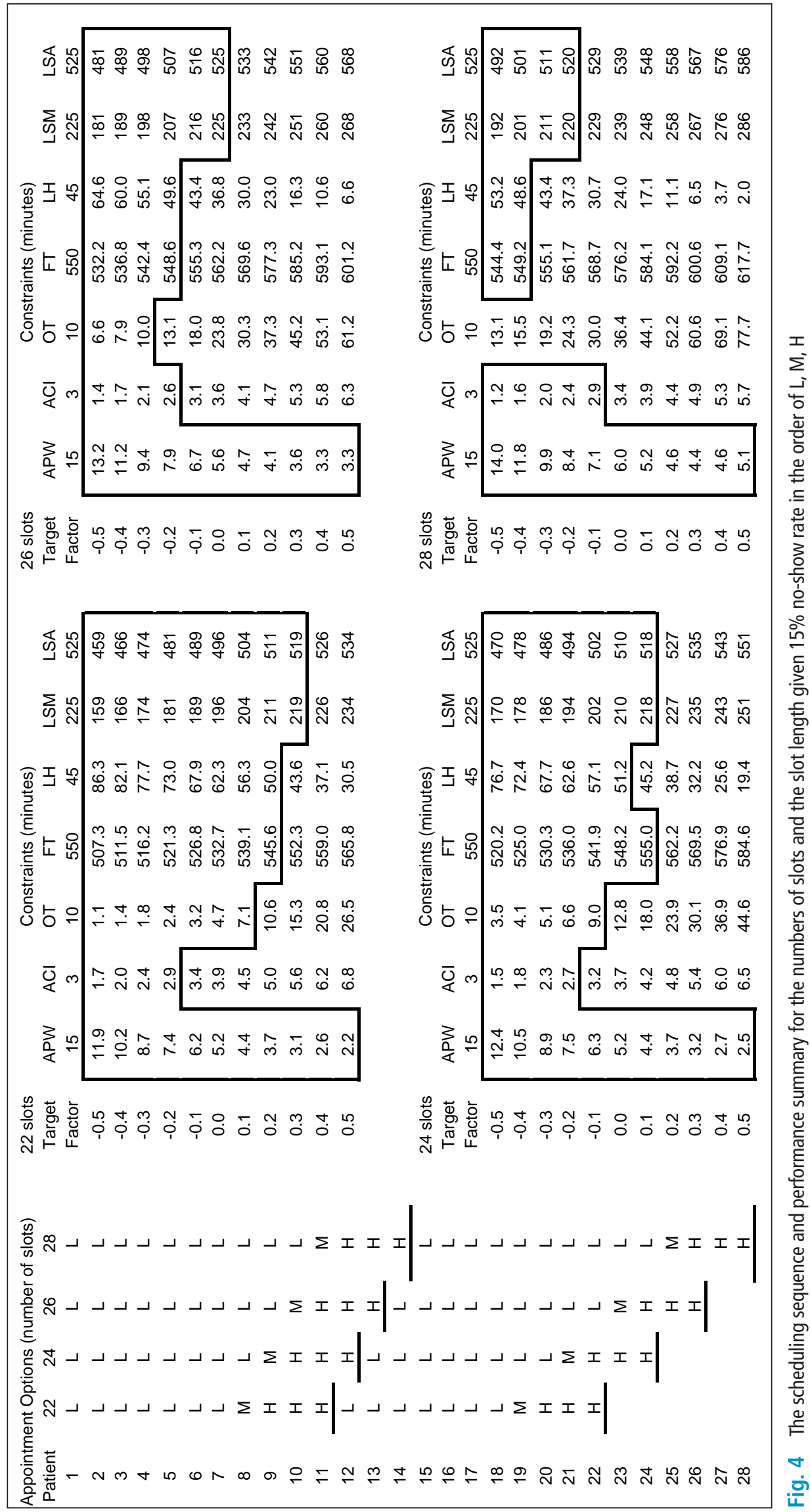




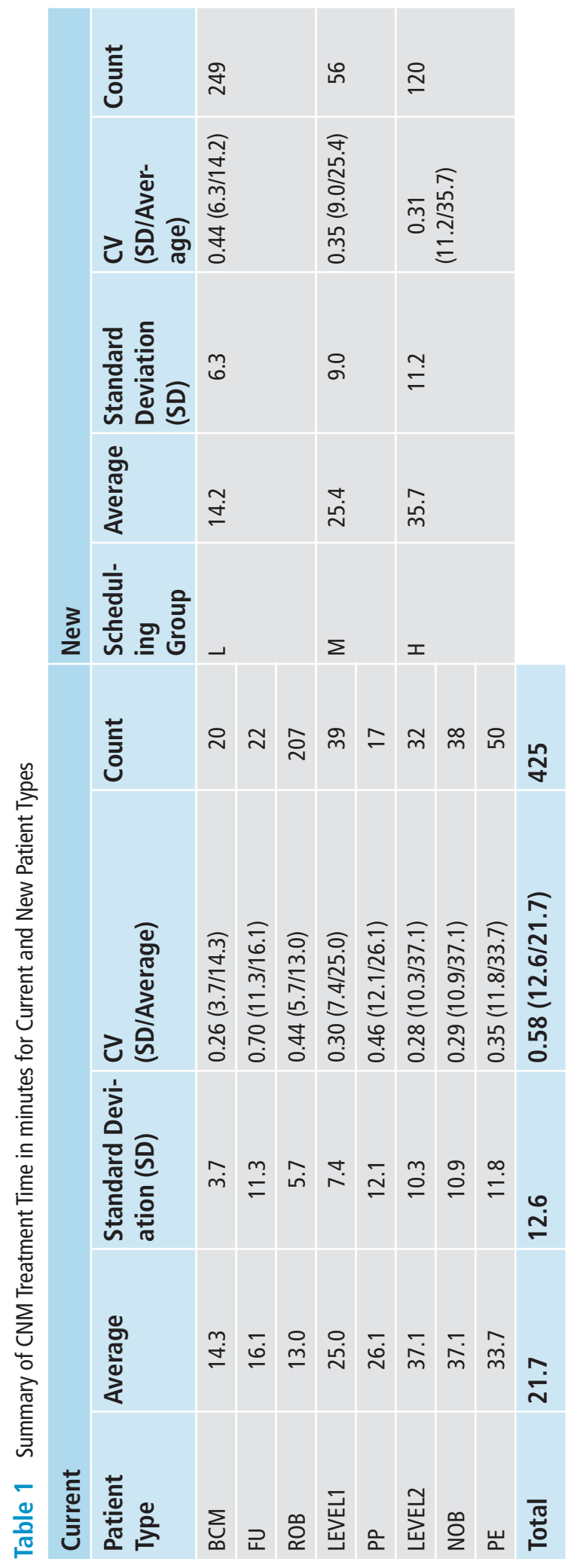


Table 2 The Minimum Volume for Determining the Number of Slots of Each Patient Type

\begin{tabular}{|l|c|l|c|c|}
\hline Current & & New & & \\
\hline Patient Type & Minimum/week & Patient Type & Target/week & Distribution \% \\
\hline ROB & 80 & L & 98 & $62.8 \%$ \\
\hline BCM & 6 & & & \\
\hline FU & 12 & & 18 & \\
\hline PP & 9 & M & & $11.5 \%$ \\
\hline Level 1 & 9 & & 40 & \\
\hline Level 2 & 7 & H & & $25.6 \%$ \\
\hline NOB & 12 & & & \\
\hline PE & 21 & & & \\
\hline
\end{tabular}

\begin{tabular}{|c|c|c|c|c|}
\hline \multicolumn{5}{|c|}{ Appointment Options (number of slots) } \\
\hline Patient & 22 & 24 & 26 & 28 \\
\hline 1 & L & $\mathrm{L}$ & $\mathrm{L}$ & L \\
\hline 2 & L & $\mathrm{L}$ & $\mathrm{L}$ & $\mathrm{L}$ \\
\hline 3 & $\mathrm{H}$ & $\mathrm{H}$ & $\mathrm{H}$ & $\mathrm{H}$ \\
\hline 4 & L & $\mathrm{L}$ & $\mathrm{L}$ & L \\
\hline 5 & M & M & M & M \\
\hline 6 & L & $\mathrm{L}$ & $\mathrm{L}$ & $\mathrm{L}$ \\
\hline 7 & L & $\mathrm{L}$ & L & L \\
\hline 8 & $\mathrm{H}$ & $\mathrm{H}$ & $\mathrm{H}$ & $\mathrm{H}$ \\
\hline 9 & L & $\mathrm{L}$ & L & $\mathrm{L}$ \\
\hline 10 & $\mathrm{~L}$ & $\mathrm{~L}$ & L & L \\
\hline 11 & $\mathrm{H}$ & $\mathrm{H}$ & $\mathrm{H}$ & $\mathrm{H}$ \\
\hline 12 & $\mathrm{~L}$ & $\mathrm{~L}$ & L & L \\
\hline 13 & L & $\mathrm{L}$ & L & $\mathrm{L}$ \\
\hline 14 & $\mathrm{H}$ & $\mathrm{L}$ & L & L \\
\hline 15 & L & $\mathrm{H}$ & L & $\mathrm{L}$ \\
\hline 16 & M & $\mathrm{L}$ & $\mathrm{H}$ & L \\
\hline 17 & L & $\mathrm{M}$ & L & $\mathrm{H}$ \\
\hline 18 & L & $\mathrm{L}$ & $\mathrm{M}$ & L \\
\hline 19 & $\mathrm{H}$ & $\mathrm{L}$ & L & $M$ \\
\hline 20 & L & $\mathrm{H}$ & $L$ & L \\
\hline 21 & L & $\mathrm{L}$ & $\mathrm{H}$ & L \\
\hline 22 & $\mathrm{H}$ & $\mathrm{L}$ & L & $\mathrm{H}$ \\
\hline 23 & & $\mathrm{H}$ & L & L \\
\hline 24 & & $\mathrm{~L}$ & $\mathrm{H}$ & L \\
\hline 25 & & & L & $\mathrm{H}$ \\
\hline 26 & & & $\mathrm{~L}$ & $\mathrm{~L}$ \\
\hline 27 & & & & L \\
\hline 28 & & & & $\mathrm{~L}$ \\
\hline
\end{tabular}

Table 3 The CNM Schedule Options based on the Number of Slots to be Evaluated 


\begin{tabular}{|l|c|c|}
\hline Patient Group & CNM Schedule & Patient Arrival Schedule \\
\hline L & $8: 00$ & $7: 35$ \\
\hline L & $8: 12$ & $7: 50$ \\
\hline H & $8: 23$ & $7: 55$ \\
\hline L & $8: 55$ & $8: 30$ \\
\hline M & $9: 06$ & $8: 40$ \\
\hline L & $9: 28$ & $9: 05$ \\
\hline L & $9: 40$ & $9: 15$ \\
\hline H & $9: 52$ & $9: 25$ \\
\hline L & $10: 23$ & $10: 00$ \\
\hline L & $10: 34$ & $10: 10$ \\
\hline H & $10: 46$ & $10: 15$ \\
\hline L & $11: 17$ & $10: 50$ \\
\hline L & $11: 29$ & $11: 05$ \\
\hline L & $1: 00$ & $12: 35$ \\
\hline L & $1: 12$ & $12: 50$ \\
\hline H & $1: 23$ & $12: 55$ \\
\hline L & $1: 55$ & $1: 30$ \\
\hline M & $2: 06$ & $1: 40$ \\
\hline L & $2: 28$ & $2: 05$ \\
\hline L & $2: 40$ & $2: 15$ \\
\hline H & $2: 52$ & $3: 00$ \\
\hline L & $3: 23$ & \\
\hline L & $3: 34$ & $3: 15$ \\
\hline H & $3: 46$ & \\
\hline
\end{tabular}

Table 4 The Finalized CNM and Patient Arrival Schedules for 26 Appointment Slots

Table 5 The Estimated Time Needed for Check-in and MA/RN Time

\begin{tabular}{|c|c|c|c|}
\hline & MA and RN time & Check-in time & Maximum time needed \\
\hline \multirow[t]{3}{*}{ L } & FU: 5-10 minutes & 5-10 minutes & \multirow[t]{3}{*}{20 minutes } \\
\hline & BCM: 7-10 minutes & 5-10 minutes & \\
\hline & ROB: $7-10$ minutes & 5-10 minutes & \\
\hline \multirow[t]{2}{*}{ M } & PP: 10-20 minutes & 5-10 minutes & \multirow[t]{2}{*}{30 minutes } \\
\hline & LEVEL 1: 5-10 minutes & 5-10 minutes & \\
\hline \multirow[t]{3}{*}{ H } & PE: 12-20 minutes & 5-10 minutes & \multirow[t]{3}{*}{30 minutes } \\
\hline & LEVEL $2: 10-15$ minutes & 5-10 minutes & \\
\hline & NOB: $15-20$ minutes & 5-10 minutes & \\
\hline
\end{tabular}




\section{References}

1. McCarthy K, McGee HM, O’Boyle CA, McCarthy K. Outpatient clinic waiting times and non-attendance as indicators of quality. Psychol Health Med 2000; 5(3): 287-293.

2. DiMino J, Blau G. The relationship between wait time after triage and show rate for intake in a nonurgent student population. J College Stud Psychother 2012; 26(3): 241-247.

3. Cayirli T, Veral E, Rosen H. Assessment of Patient Classification in Appointment System Design. Prod Oper Manag. 2008;17(3):338-53.

4. Wijewickrama A, Takakuwa S. Designing Outpatient Appointment Systems with Patient Characteristics: a Case Study. Int J of Healthcare Technology and Management 2012; 13(1/2/3): 157-169.

5. Huang Y, Kammerdiner A. Reduction of service time variation in patient visit groups using decision tree method for an effective scheduling. Int J of Healthcare Technology and Management 2013; 14(1/2): 3-21.

6. Huang Y, Marcak J. Radiology scheduling with consideration of patient characteristics to improve patient access to care and medical resource utilization. Health Systems 2013; 2(2): 93-102.

7. Erdogan SA, Denton B. Dynamic Appointment Scheduling of a Stochastic Server with Uncertain Demand. J Comput. 2013;25(1):116-32.

8. Schutz HJ, Kolisch R. Capacity allocation for demand of different customer-product-combinations with cancellations, no-shows, and overbooking when there is a sequential delivery of service. Ann Oper Res 2013; 206(1): 401-423.

9. Hahn-Goldberg S, Carter MW, Beck JC, Trudeau M, Sousa P, Beattie K. Dynamic optimization of chemotherapy outpatient scheduling with uncertainty. Health Care Manage Sci DOI 10.1007/s10729-014-9268-0.

10. Tang J, Yan C, Cao P. Appointment scheduling algorithm considering routine and urgent patients. Expert Syst Appl 2014; 41(10): 4529-4541.

11. Huang Y, Zuniga P, Marcak J. A cost-effective urgent care policy to improve patient access in a dynamic scheduled clinic setting. J Oper Res Soc 2014; 65(5): 763-776.

12. Chen RR, Robinson LW. Sequencing and Scheduling Appointments with Potential Call-In Patients. Prod Oper Manag 2014; 23(9): 1522-1538.

13. Tai G, Williams P. Optimization of scheduling patient appointments in clinics using a novel modeling technique of patient arrival. Comput Meth Prog Bio 2011; 108(2): 467-476.

14. Klassen KJ, Yoogalingam R. Appointment system design with interruptions and physician lateness. Int J Oper Prod Man 2013; 33(4): 394-414.

15. Cayirli T, Yang KK, Quek SA. A Universal Appointment Rule in the Presence of No-Shows and Walk-Ins. Prod Oper Manag 2012; 21(4): 682-697.

16. Huang Y. Ancillary service impact on outpatient scheduling. Int J Health Care Qual Assur 2013; 26(8): 746-759.

17. Huang Y, Zuniga P. Dynamic overbooking scheduling system to improve patient access. J Oper Res Soc 2012; 63(6): 810-820.

18.LaGanga LR, Lawrence SR. Appointment Overbooking in Health care Clinics to Improve Patient Service and Clinic Performance. Prod Oper Manag 2012; 21(5): 874-888.

19.Zeng B, Zhao H, Lawley M. The impact of overbooking on primary care patient no-show. IIE Trans Healthc Syst Eng 2013; 3(3): 147-170.

20.Zacharias C, Pinedo M. Appointment Scheduling with No-Shows and Overbooking. Prod Oper Manag 2014; 23(5): 788-801.

21.Tsai PJ, Teng G. A stochastic appointment scheduling system on multiple resources with dynamic call-in sequence and patient no-shows for an outpatient clinic. Eur J Oper Res 2014; 239(2): 427-436.

22. Huang Y, Hanauer DA. Patient No-Show Predictive Model Development using Multiple Data Sources for an Effective Overbooking Approach. Appl Clin Inform 2014; 5(3): 836-860.

23. Samorani M, LaGanga LR. Outpatient appointment scheduling given individual day-dependent no-show predictions. Eur J Oper Res 2015; 240(1): 245-257.

24.Kim S, Giachetti RE. A Stochastic Mathematical Appointment Overbooking Model for Healthcare Providers to Improve Profits. IEEE Trans Syst Man Cybern 2006; 36(6): 1211-1219.

25. Berg BP, Denton BT, Erdogan SA, Rohleder T, Huschka T. Optimal booking and scheduling in outpatient procedure centers. Comput Oper Res 2014; 50: 24-37.

26. Vanden Bosch PM, Dietz DC. Minimizing expected waiting in a medical appointment system. IIE Trans 2000; 32(9): 841-848.

27.Harper PR, Gamlin HM. Reduced outpatient waiting times with improved appointment scheduling: a simulation modeling approach. OR Spectrum 2003; 25(2): 207-222. 
28. Huang Y, Hancock WM, Herrin GD. An alternative outpatient scheduling system: Improving the outpatient experience. IIE Trans Healthc Syst Eng 2012; 2(2): 97-111.

29. OH H, Muriel A, Balasubramanian H, Atkinson K, Ptaszkiewicz T. Guidelines for scheduling in primary care under different patient types and stochastic nurse and provider service times. IIE Trans Healthc Syst Eng 2013; 3(4): 263-279.

30. Kuiper A, Kemper B, Mandjes M. A Computational approach to optimized appointment scheduling. Queueing Systems. DOI 10.1007/s11134-014-9398-6.

31. Keller TF, Laughhunn DJ. An Application of Queuing Theory to a Congestion Problem in an Outpatient Clinic. Decision Sci 1973; 4(3): 379-394.

32. Yang KK, Lau ML, Quek SA. A New Appointment Rule for a Single-Server, Multiple-Customer Service System. Nav Res Log 1998; 45(3): 313-326.

33. O'Connor ME, Matthews BS, Gao D. Effect of Open Access Scheduling on Missed Appointments, Immunizations, and Continuity of Care for Infant Well-Child Care Visits. Arch Pediat Adol Med 2006; 160(9): 889-893.

34. Bundy DG, Randolph GD, Murray M, Anderson J, Margolis PA. Open Access in Primary Care: Results of a North Carolina Pilot Project Pediatrics 2005; 116(1): 82-87.

35. Forjuoh SN, Averitt WM, Cauthen DB, Couchman GR, Symm B, Mitchell M. Open-Access Appoinment Scheduling in Family Practice: Comparison of a Demand Prediction Grid With Actual Appointments. J Am Board Fam Pract 2001; 14(4): 259-265.

36. Peng Y, Qu X, Shi J. A hybrid simulation and genetic algorithm approach to determine the optimal scheduling templates for open access clinics admitting walk-in patients. Comput Ind Eng 2014; 72: 282-296

37. Lee S, Min D, Ryu J, Yih Y. A simulation study of appointment scheduling in outpatient clinics: Open access and overbooking. Simulation 2013; 89(12): 1459-1473.

38. Klassen KJ, Rohleder TR. Scheduling outpatient appointments in a dynamic environment. J Oper Manag 1996; 14(2): 83-101.

39. Cayirli T, Veral E, Rosen H. Designing appointment scheduling systems for ambulatory care services. Health Care Manage Sci 2006; 9(1): 47-58. 